\title{
OCORRÊNCIA DE MOVIMENTOS PRIMÁRIOS DE LÍNGUA EM CRIANÇAS RESPIRADORAS ORONASAIS
}

\author{
Occurrence of primary tongue movements \\ in children with oronasal breathing
}

\author{
Cássia Sígolo ${ }^{(1)}$, Michelly Silveira ${ }^{(2)}$, Maíra Quintal ${ }^{(3)}$, Eulália Sakano ${ }^{(4)}$, Adriana Tessitore ${ }^{(5)}$
}

\begin{abstract}
RESUMO
Objetivo: quantificar a ocorrência de movimentos primários de língua em 130 crianças respiradores oronasais e em 89 respiradoras nasais, com idades de cinco a 12 anos, avaliadas no Setor de Respiração Oral do Ambulatório de Otorrinolaringologia do Hospital de Clínicas da Universidade Estadual de Campinas. Métodos: no momento da avaliação fonoaudiológica, foi solicitado à criança deglutir saliva, fechar os olhos, colocar a língua para fora da boca e permanecer assim por, no mínimo, um minuto, tempo necessário para os movimentos primários serem observados. Duas fonoaudiólogas observaram juntas cada criança, a fim de constatar a presença dos movimentos ou não. Resultados: sobre a ocorrência de movimentos primários de língua, foi encontrada em $44,75 \%$ da amostra $(n=98)$, enquanto $55,25 \%$ ( $n=121)$ não apresentaram tais movimentos. Foi observado que as categorias, nasal e oronasal, apresentaram diferenças estatisticamente para a ocorrência de movimentos primários de língua. Conclusão: em respiração oronasal há uma maior freqüência de manutenção dos movimentos primários de língua e há diferença estatisticamente significante entre o grupo de respiradores oronasais e respiradores nasais para a variável movimento primário de língua.
\end{abstract}

DESCRITORES: Respiração Bucal; Criança; Língua; Força Muscular; Deglutição

(1) Fonoaudióloga do Setor de Fonoaudiologia do Ambulatório de Respiração Oral da Disciplina de Otorrinolaringologia do Hospital das Clínicas da Universidade Estadual de Campinas; Aperfeiçoamento em Motricidade Orofacial pela Irmandade da Santa Casa de Misericórdia de São Paulo; Mestre em Saúde da Criança e do Adolescente pela Universidade Estadual de Campinas.

(2) Fonoaudióloga do Setor de Fonoaudiologia do Ambulatório de Respiração Oral da Disciplina de Otorrinolaringologia do Hospital das Clínicas da Universidade Estadual de Campinas; Terapeuta no Conceito de Reabilitação Orofacial e Corporal Castillo-Morales; Aperfeiçoamento em Disfagia Neonatal pela Irmandade da Santa Casa de Misericórdia de São Paulo.

(3) Fonoaudióloga do Setor de Fonoaudiologia do Ambulatório de Respiração Oral da Disciplina de Otorrinolaringologia do Hospital das Clínicas da Universidade Estadual de Campinas; Terapeuta no Conceito de Reabilitação Orofacial e Corporal Castillo-Morales; Especialista em Motricidade Orofacial pelo CEFAC - Saúde e Educação.

(4) Médica Otorrinolaringologista; Chefe do Ambulatório de Respiração Oral do Hospital das Clínicas da Universidade Estadual de Campinas; Doutora em Ciências Médicas pela Universidade Estadual de Campinas.

(5) Fonoaudióloga Responsável pelo Setor de Fonoaudiologia do Ambulatório de Respiração Oral da Disciplina de Otorrinolaringologia do Hospital das Clínicas da Universidade Estadual de Campinas; Professora do CEFAC - Saúde e Educação; Especialista em Motricidade Orofacial; Doutoranda em Ciências Médicas pela Universidade Estadual de Campinas.

\section{INTRODUÇÃO}

A respiração oral é um sintoma freqüente na infância ${ }^{1}$ e 0 número de pacientes respiradores orais vem aumentando gradativamente em função da poluição, e conseqüentemente, do aumento dos agentes alérgicos, cada vez mais presentes no ar e nos alimentos ${ }^{2}$. Por isso, muitos pacientes chegam ao consultório fonoaudiológico encaminhados por vários profissionais da área da saúde como otorrinolaringologistas, dentistas, alergistas, pediatras, fisioterapeutas e psicólogos, e há um consenso da necessidade de uma equipe interdisciplinar para a eficiência no tratamento devido a sua complexidade $2-4$.

Existem diversos fatores que levam a criança a respirar pela boca. A presença de uma obstrução nasal, que pode ser causada por um quadro de rinite, rinosinusopatia, hipertrofia e inflamação das tonsilas palatinas e faríngeas, desvio do septo nasal, dilatação das conchas ou hipertrofia da membrana da mucosa nasal, dentre outras causas prováveis, poderá gerar a respiração oral como uma função adaptativa do sistema estomatognático ${ }^{5}$. 
Dentre os problemas respiratórios, a rinite alérgica e as hipertrofias de amígdalas e/ou adenóides são as mais encontradas nas crianças em fase de crescimento ${ }^{6}$.

Além disso, há fatores de natureza não-obstrutivas que podem contribuir para o surgimento de uma respiração oral, como: hábitos orais deletérios, amamentação materna insuficiente, hábito de sucção de polegar ou de chupeta e malformações craniofaciais (alterações da mandíbula, de língua e hipotonicidade muscular generalizada) ${ }^{7}$.

O otorrinolaringologista é o profissional que avalia as condições anatomofuncionais da cavidade nasal ${ }^{8}$, a fim de verificar se o tipo respiratório é nasal (respiração realizada pelo nariz); oral (respiração realizada predominantemente pela boca) ou oronasal (respiração realizada pela boca e pelo nariz) ${ }^{9}$.

A respiração oral não deve ser considerada como uma alternativa à dificuldade de respirar pelo nariz, mas sim como uma adaptação patológica que pode resultar em inúmeras alterações para o indivíduo ${ }^{10,11}$.

Estas possíveis alterações encontradas em respiradores orais podem ser classificadas em alterações craniofaciais e dentárias ${ }^{12}$; dos órgãos fonoarticulatórios e corporais; das funções orais e alterações médicas e otorrinolaringológicas ${ }^{13,14}$.

Algumas alterações dos órgãos fonoarticulatórios que podem ocorrer em respiradores orais são: hipotonia, hipotrofia e hipofunção dos músculos elevadores da mandíbula; alteração dos tônus muscular dos lábios e bochechas; tensão do músculo constritor da faringe ${ }^{4}$; hipotonia facial generalizada; músculos supra-hióideos tensos; lábio inferior interposto entre os dentes ou evertido; retração ou encurtamento do lábio superior; lábios com alteração de cor, secos, fissurados e feridos; frênulo labial curto ${ }^{14}$; posição habitual da língua alterada; alteração da sensibilidade e coloração na mucosa lingual, labial e das bochechas; fibras musculares marginais com encurtamento; face assimétrica; ângulo nasal obtuso; má configuração ou tensão do sulco nasogeniano; diminuição da pressão intra-oral e presença de movimentos primários de língua ${ }^{8,15-17}$.

Em estudos realizados ${ }^{18,19}$ entre 1995 a 1998 em crianças com lesões cerebrais, Síndrome de Down e com outras patologias genéticas acompanhadas de hipotonia, foi descoberta a existência de movimentos espontâneos de língua. Estes foram observados ao abrir a boca do paciente e notou-se a ocorrência de movimentos espontâneos e involuntários, que se repetiam sempre e se apresentavam de forma igual. Inicialmente tais movimentos eram chamados pelos autores Castillo Morales e Molina ${ }^{18,19}$ de "movimentos com componentes atetóides", presumindo-se que eram produzidos por uma incoordenação central. Em outra pesquisa, foram estudadas crianças prematuras até seis anos de idade, sem alterações neurológicas, e constatou-se que os mesmos movimentos estavam presentes ${ }^{18}$. A partir de estudo ${ }^{16}$, Castillo Morales denominou estes movimentos espontâneos e involuntários, que só aconteceu com a língua em repouso, como movimentos primários de língua (MPL).

Os MPL são movimentos próprios e involuntários da língua, simétricos, que aumentam antes da alimentação; além disso, são descritos como fisiológicos (mecanismo necessário de todo mamífero) e coincidem com o aparecimento de movimentos de sucção e deglutição ${ }^{8,16-19}$.

Os MPL são estereotipados, visíveis quando ela se movimenta espontaneamente com a boca aberta e tornam-se mais evidentes quando a língua está fora da boca. Para serem observados, a língua deve ficar relaxada fora da boca. Os movimentos começam em sua raiz, avançam em forma de ondas em direção ao ápice e aos poucos, desmancham-se. Posteriormente, ocorre uma pequena contração nas margens laterais da língua no sentido da linha mediana; a língua afunila-se e no centro forma-se uma leve elevação que desaparece imediatamente e depois é seguida por um abaixamento em sua parte medial. Os movimentos são simétricos e rítmicos, e os movimentos de elevação e abaixamentos da parte média da língua alternam-se ${ }^{20}$. Tais movimentos ocorrem póstero-anteriormente, como uma onda peristáltica, de baixo para cima, com uma contração medial e terminam em forma de colher ${ }^{8,20}$; são imprescindíveis para a alimentação, pois a criança os apresenta desde o nascimento e, ao iniciar a sucção, estes movimentos dirigem-se para trás e possibilitam o impulso posterior do alimento.

Os movimentos vão diminuindo gradativamente quando o mecanismo de sucção passa a ser voluntário, e desaparecem aos quatro anos de idade, coincidindo com o desenvolvimento de funções motoras finas, corporais e manuais ${ }^{16}$.

Os fatores que incidem na persistência de movimentos primários de língua depois dos cinco anos de idade foram obtidos em um estudo sobre a persistência de movimentos primários de língua em grupos de crianças de cinco anos de idade sem patologia neurológica, e são: uso de chupeta até os 18 meses de idade; sucção de polegar ou de outro elemento; hábitos de interposição lingual e/ou sucção digital; alterações dentárias; deglutição com padrão infantil e respiração oral ${ }^{8,16}$.

A pesquisa ${ }^{16}$ também demonstrou, entre outros aspectos, que há uma incidência significativa de alterações na produção do fone vibrante simples [r] em 
posição de consoante vogal como em -barata-, assim como em posição de grupo consonantal como em -prato- em crianças onde os MPL persistiram após os quatro anos de idade.

Mediante estes dados levantados na literatura e pela pouca quantidade de estudos realizados sobre os movimentos primários de língua, o objetivo deste estudo foi verificar e quantificar a ocorrência de MPL em 130 casos de respiradores oronasais (RON) e 89 casos de respiradores nasais (RN), com idades de cinco a 12 anos, avaliadas no Setor de Respiração Oral do Ambulatório de Otorrinolaringologia do Hospital de Clínicas da Universidade Estadual de Campinas.

\section{MÉTODOS}

O presente estudo observacional descritivo foi realizado com 130 crianças $R O N$ e $89 R N$, na faixa etária de cinco a 12 anos, que passaram por avaliação no Setor de Fonoaudiologia do Ambulatório de Respiração Oral, da Disciplina de Otorrinolaringologia do Hospital de Clínicas da Universidade Estadual de Campinas, no período de maio de 2005 a fevereiro de 2006.

A idade das crianças participantes correspondeu às exigências de atendimento do Setor de Fonoaudiologia; eram de ambos os sexos, independente de sua etnia e de classe sócio-econômico-cultural.

Os critérios de inclusão foram: faixa etária entre cinco a 12 anos de idade, apresentar respiração oronasal e ter sido avaliado por otorrinolaringologista.

Os sujeitos foram classificados como RN ou RON pelos médicos otorrinolaringologistas do Setor.

No momento da avaliação fonoaudiológica a criança estava sentada em cadeira, encostada no espaldar, com pernas e coluna em $90^{\circ}$, pés apoiados no chão ou em uma escada, caso não conseguisse alcançá-los no chão, mãos apoiadas nos joeIhos. Foi solicitado à criança deglutir saliva, fechar os olhos, colocar a língua para fora da boca e permanecer assim por, no mínimo, um minuto, tempo necessário para os movimentos primários serem observados. Duas fonoaudiólogas observaram juntas cada criança, a fim de constatar a presença ou não dos movimentos.

O tempo foi marcado com cronômetro e o registro da presença ou ausência dos movimentos foi realizado em um item no próprio protocolo de triagem fonoaudiológica utilizado no Setor (Figura 1).

Para documentar este procedimento foi utilizada a câmera filmadora Sony DCR-HC28 fixada em um tripé a uma distância de 1,0 metro do paciente. 0 enquadramento da filmagem foi feito no terço inferior de sua face. Não foi utilizado zoom.
O Comitê de Ética em Pesquisa do CEFAC Saúde e Educação avaliou e aprovou esta pesquisa sob o número 113/05 e considerou sem risco e com necessidade de consentimento pós-informado.

Em relação à análise estatística, foi adotado o nível de significância de $5 \%(0,050)$, para a aplicação dos testes estatísticos e utilizado o programa SPSS (Statistical Package for Social Sciences), em sua versão 13.0, para a obtenção dos resultados.

Em seguida, foi aplicado o Teste $t$ de Student, controlado pelo Teste de Levene para Igualdade de Variâncias, com o intuito de verificarem-se possíveis diferenças entre as duas categorias da variável 'respiração', para variável paramétrica e o Teste de Mann-Whitney, com o intuito de verificarem-se possíveis diferenças entre as duas categorias da variável 'respiração' para variáveis não-paramétricas.

\section{RESULTADOS}

Nesta amostra de 219 casos, a média de idade foi de 6,10 anos, conforme Tabela 1.

Sobre as variáveis não paramétricas, observouse que $43,84 \%(n=96)$ eram do sexo feminino e $56,16 \%(n=123)$ eram do masculino; $59,36 \%(n=130)$ apresentavam respiração oronasal e 40,64\% ( $n=89)$ eram respiradores nasais e, sobre a ocorrência de MPL, em $44,75 \%$ da amostra $(n=98)$ foi encontrada a presença de MPL, enquanto $55,25 \%(n=121)$ não apresentaram tais movimentos (Tabelas 2, 3 e 4, respectivamente).

Para a comparação entre os dois tipos de respiração para a variável paramétrica 'idade', foi aplicado o Teste $t$ de Student, controlado pelo Teste de Levene para Igualdade de Variâncias, com o intuito de verificarem-se possíveis diferenças entre as duas categorias da variável 'respiração', e o valor de $p$ encontrado foi $p<0,001$.

\section{Tabela 1 - Distribuição dos casos quanto à idade, em anos ( $n=219)$}

\begin{tabular}{cccccc}
\hline Variável & $\mathbf{n}$ & \multicolumn{2}{c}{ Mínimo Máximo } & Média & $\begin{array}{c}\text { Desvio- } \\
\text { padrão }\end{array}$ \\
\hline Idade & 219 & 6,00 & 12,00 & 6,10 & 3,20 \\
\hline
\end{tabular}

Legenda: (n): freqüência absoluta

Tabela 2 - Distribuição dos casos quanto ao sexo $(n=219)$

\begin{tabular}{ccc}
\hline Gênero & Freqüência & Percentual \\
\hline feminino & 96 & 43,84 \\
masculino & 123 & 56,16 \\
Total & 219 & 100,00 \\
\hline
\end{tabular}


Universidade Estadual de Campinas - UNICAMP

Ambulatório de Otorrinolaringologia

Setor de Fonoaudiologia / Respiração Oral

UNICAMP

Triagem Fonoaudiológica

Informante:

Data:

Nome:

$\mathrm{HC}$ :

Data de Nascimento:

Idade:

Escolaridade:

Endereço:

Telefone:

Queixa:

Alimentação atual:

$\begin{array}{lll}\text { Consistente: } & \text { ( ) sim ( ) não Ritmo: ( ) devagar } \text { ( ) rápido }_{\text {Usa líquido: }} & \text { ( ) sim ( ) não Come de boca aberta: ( ) sim adequado }\end{array}$

Observações:

Hábitos:

Sucção: mamadeira ( ) chupeta ( ) dedo ( ) Idade:

Onicofagia ( ) Mordiscar objetos: ( ) Quais?

Sialorréia: ( ) diurna ( ) noturna Outros:

Ambiente:

Quarto: ( ) cortina ( ) tapete ( ) objetos de pelúcia / outros ( ) cobertor

Quintal: ( ) terra ( ) animal de estimaçao - Qual?

Rua: ( ) terra ( ) asfalto ( ) fumante na casa

Observações:

Aspecto geral dos OFAs / Funções Orais:

\begin{tabular}{|c|c|c|c|}
\hline Lábios: & ( ) entreabertos & $\begin{array}{l}\text { ( ) superior } \\
\text { encurtado }\end{array}$ & $\begin{array}{l}\text { ( ) inferior } \\
\text { evertido }\end{array}$ \\
\hline Hipercontração mentual: & ( ) presente & ( ) ausente & \\
\hline Movimentos primários de língua: & ( ) presentes & ( ) ausentes & \\
\hline Interposição linguo-dental: & ( ) presente & ) ausente & \\
\hline Bochechas: & ) simétricas & assimétricas & \\
\hline Narinas: & ) simétricas & ) assimétricas & \\
\hline Tipo facial: & curto & longo & \\
\hline Palato: & alto & normal & \\
\hline Respiração: & ) nasal & oral & ( ) oronasal \\
\hline Mastigação: & normal & ( ) alterada & \\
\hline Deglutição: & ( ) normal & ( ) atípica & ( ) adaptada \\
\hline Hábitos parafuncionais: & ( ) presentes & ( ) ausentes & \\
\hline Fala: & ( ) normal & ( ) alterada & \\
\hline Voz: & ( ) adequada & ( ) hipernasal & ( ) hiponasal \\
\hline
\end{tabular}

Conclusão:

Conduta:

Fga. Responsável:

Fga. Responsável:

Figura 1 - Protocolo utilizado na triagem fonoaudiológica no Setor de Fonoaudiologia do Ambulatório de Otorrinolaringologia da Universidade Estadual de Campinas

Rev CEFAC, São Paulo, v.10, n.1, 51-57, jan-mar, 2008 
Tabela 3 - Distribuição dos casos quanto ao tipo respiratório $(n=219)$

\begin{tabular}{ccc}
\hline respiração & Freqüência & Percentual \\
\hline oronasal & 130 & 59,36 \\
nasal & 89 & 40,64 \\
Total & 219 & 100,00 \\
\hline
\end{tabular}

Tabela 4 - Distribuição dos casos quanto à ocorrência de MPL $(n=219)$

\begin{tabular}{ccc}
\hline MPL & Freqüência & Percentual \\
\hline presentes & 98 & 44,75 \\
ausentes & 121 & 55,25 \\
Total & 219 & 100,00 \\
\hline
\end{tabular}

Tabela 5 - Correlação entre tipo respiratório e idade

\begin{tabular}{cccccc}
\hline Variável & respiração & $\mathbf{n}$ & Média & Desvio-padrão & Significância $(\mathbf{p})$ \\
\hline \multirow{2}{*}{ idade } & oronasal & 130 & 4,18 & 2,15 & \multirow{2}{*}{0,001} \\
\cline { 2 - 5 } & nasal & 89 & 8,90 & 2,27 & \\
\hline
\end{tabular}

A aplicação do Teste de Mann-Whitney foi realizada com o intuito de verificarem-se possíveis diferenças entre as duas categorias da variável 'respiração' para as variáveis não-paramétricas 'gênero' e 'ocorrência de MPL', sendo que para 'gênero', $p=0,098$ (Tabela 5) e para MPL, $p<0,001$ (Tabela 6).

Foi observado que as categorias nasal e oronasal apresentam diferenças estatisticamente para as variáveis 'idade' e 'MPL', conforme o resultado apurado pelas análises estatísticas, acima (Tabelas 5 e 6).

\section{Tabela 6 - Correlação entre tipo respiratório} e sexo

\begin{tabular}{cccc}
\hline \multirow{2}{*}{ respiração } & \multicolumn{2}{c}{ gênero } & \multirow{2}{*}{ Total } \\
\cline { 2 - 3 } & feminino & masculino & \\
\hline \multirow{2}{*}{ oral } & 51 & 79 & 130 \\
& 39,23 & 60,77 & 100,00 \\
\hline \multirow{2}{*}{ nasal } & 45 & 44 & 89 \\
& 50,56 & 49,44 & 100,00 \\
\hline \multirow{2}{*}{ Total } & 96 & 123 & 219 \\
& 43,84 & 56,16 & 100,00 \\
\hline
\end{tabular}

$p=0,098$

\section{DISCUSSÃO}

Ao comparar os dados deste trabalho com um estudo sobre a Persistência dos Movimentos Primários de Língua e sua ocorrência na articulação da fala, realizado na Argentina em $1996{ }^{16}$, foi observado que, de 27 crianças com cinco anos de idade, 29,63\% apresentavam respiração nasal, $44,44 \%$ respiração oral e $25,93 \%$ respiração orona- sal; de 26 crianças com seis anos de idade, 42,31\% apresentavam respiração nasal, 26,92\% respiração oral e $30,77 \%$ respiração oronasal. Neste estudo, a maioria das crianças apresentou respiração oronasal $(n=130)$.

No presente estudo, em que foi utilizado somente casos de RON ( $n=130)$ e RN $(n=89)$, foi encontrada a presença dos movimentos primários de língua em $59,23 \%(n=77)$ dos $R O N$ e em $23,60 \%(n=21)$ dos RN, demonstrando o que a literatura sugere que existe alguma relação entre a alteração do modo respiratório com a manutenção destes movimentos ${ }^{18,19}$.

Esta pesquisa foi um estudo preliminar onde se estudou em primeiro plano se realmente em RON estes movimentos são preservados ou não. Será dada continuidade neste estudo levantando a ocorrência ou não de alterações na fala, como sugere a literatura ${ }^{16}$.

A avaliação fonoaudiológica em respiradores orais deve envolver as funções estomatognáticas (sucção, mastigação, deglutição e fonação) ${ }^{15}$, um exame detalhado das estruturas do complexo orofacial (lábios, língua, palato mole e duro, dentes, gengiva, articulação temporomandibular, mandíbula, maxila e oclusão), a fim de relacionar a forma à função ${ }^{21-24}$, além da documentação fotográfica das estruturas orais e da postura corporal ${ }^{25} \mathrm{e}$ da documentação funcional.

Devido à ocorrência de MPL em crianças RON, sugere-se o acréscimo deste item na avaliação fonoaudiológica, o que possibilitará em avaliação e processo terapêutico mais fidedignos, além de nos proporcionar a reflexão sobre o limite terapêutico que estes movimentos podem causar, relacionados à fala, recidivas de tratamento odontológico ${ }^{16}$. 
A presença de movimentos primários de língua é um dado muito importante para o planejamento terapêutico do fonoaudiólogo, pois se estes forem constatados, deve-se levar em consideração como fator prognóstico visto que há uma incidência de alteração da qualidade na produção de determinados fones como o vibrante simples [r] em posição de consoante vogal e de grupo consonantal.

Para o futuro seria de grande valia novos estudos sobre a presença e possíveis conseqüências advindas da presença de movimentos primários de língua, como as poucas pesquisas nesta área já conseguiram constatar.

Apesar da pouca literatura sobre o tema, buscou-se aqui avaliar se a respiração oronasal é de fato um fator que favorece a manutenção dos MPL, e observou-se que realmente há uma incidência

\section{Tabela 7 - Correlação entre tipo respiratório e ocorrência de MPL}

\begin{tabular}{|c|c|c|c|}
\hline \multirow{2}{*}{ respiração } & \multicolumn{2}{|c|}{ MPL } & \multirow{2}{*}{ Total } \\
\hline & presente & ausente & \\
\hline \multirow{2}{*}{ oral } & 77 & 53 & 130 \\
\hline & 59,23 & 40,77 & 100,00 \\
\hline \multirow{2}{*}{ nasal } & 21 & 68 & 89 \\
\hline & 23,60 & 76,40 & 100,00 \\
\hline \multirow{2}{*}{ Total } & 98 & 121 & 219 \\
\hline & 44,75 & 55,25 & 100,00 \\
\hline
\end{tabular}

$\mathrm{p}<0,001$ maior nos RON em relação aos RN (Tabela 7), confirmando os dados da literatura ${ }^{18,19}$.

Outro aspecto avaliado no grupo controle de RN que apresentavam a MPL, foi que todos estes $(n=21)$ também apresentaram alteração na fala no fone vibrante simples (Tabela 8 ), o que justifica a investigação de alteração de fala nos RON que apresentam MPL, pois pode fazer com que a Fonoaudiologia considere este dado clínico como fator prognóstico negativo em casos com estas características, justificando nestes casos limites estruturais para uma articulação perfeita da fala.

\section{Tabela 8 - Distribuição dos RN quanto à alteração de fala $(n=89)$}

\begin{tabular}{cccc}
\hline \multirow{2}{*}{ respiração } & \multicolumn{3}{c}{ Alteração de fala } \\
\cline { 2 - 4 } & presente & ausente & total \\
\hline \multirow{3}{*}{ nasal } & 22 & 67 & 89 \\
& 24,72 & 75,28 & 100,00 \\
& 10,05 & 30,59 & 100,00 \\
\hline
\end{tabular}

\section{CONCLUSÃO}

Concluiu-se que em respiradores oronasais há uma maior freqüência de manutenção dos movimentos primários da língua (MPL) e que há diferença estatisticamente significante entre o grupo de respiradores oronasais e respiradores nasais para a variável MPL.

\section{ABSTRACT}

Purpose: to quantify the occurrence of primary tongue movements in 130 children with oronasal breathing and in 89 with nasal breathing with ages between 5 and 12 years old, evaluated in the Section of Mouth Breathing, of the Clinical Hospital of Unicamp, within the Otorrinolaringology Clinic. Methods: during the evaluation, the children were requested to swallow saliva, close their eyes and put their tongue outside the mouth. They were supposed to stay like this for, at least, one minute, which is enough time to monitor their primary movements. Two language speech pathologists monitored each child, in order to verify the presence of movements. Results: the occurrence of the primary tongue movements was found in $44.75 \%$ of the sample $(n=98)$, while $55.25 \%(n=121)$ did not show any such movements. It was observed that both nasal and oronasal categories showed statistically significant difference as for the occurrences of primary tongue movements. Conclusion: oronasal breathing has higher frequency for maintenance of primary tongue movements, and there is statistically significant difference between oronasal and nasal breathing groups as for the primary tongue movement's variable.

KEYWORDS: Mouth Breathing; Child; Tongue; Muscle Strength; Deglutition 


\section{REFERÊNCIAS}

1. Di Francesco RC, Passeroti G, Paulucci B, Miniti A. Respiração oral na criança: repercussões diferentes de acordo com o diagnóstico. Rev Bras Otorrinolaringol. 2004; 70(5):665-70.

2. Silva HJC, Fontes ML, Paixão C, Maciel A. Reeducação Postural Global (RPG): contribuições ao paciente respirador oral em fonoterapia. Rev Soc Bras Fonoaudiol. 2002; 7(2):53-9.

3. Miranda PPC, Mashuda SYK, Periotto MC, Araújo RJH. Enfoque multidisciplinar na síndrome do respirador bucal. Rev Paul Odontol. 2002; 24(3):4-8.

4. Parolo AMF, Bianchini EMG. Pacientes portadores de respiração bucal: uma abordagem fonoaudiológica. Rev Dental Press Ortodon Ortop Facial. 2000; 5(2):76-81.

5. Campiotto AR, Reis DM, Gaeta RT. Princípios da intervenção fonoaudiológica nos distúrbios miofuncionais orofaciais. In: Filho OL, organizador. Tratado de fonoaudiologia. São Paulo: Tecmedd; 2004. p. $735-40$.

6. Faria PT, Ruelas ACO, Matsumoto MA, AnselmoLima WT, Pereira FC. Dentofacial morphology of mouth breathing children. Braz Dent J. 2002; 13(2):129-32.

7. Barros JR, Becker HM, Pinto JA. Evaluation of atopy among mouth-breathing pediatric patients referred for treatment to a tertiary care center. J Pediatr. 2006; 82(6):458-64.

8. Tessitore A. Alterações oromiofuncionais em respiradores orais. In: Ferreira LP, Befi-Lopes DM, Limongi SCO, organizador. Tratado de fonoaudiologia. São Paulo: Roca; 2004. p. 261-76.

9. Duarte LIM, Krakauer LH, Cattoni DM, organizador. Documento oficial 04/2007 do Comitê de Motricidade Orofacial da Sociedade Brasileira de Fonoaudiologia. São Paulo; 2007.

10. Di Francesco RC. Definindo a respiração oral. In: Krakauer L, Di Francesco RC, Macchesan I, organizador. Respiração oral: abordagem multidisciplinar. São José dos Campos: Pulso; 2003. p.15-7.

11. Parra Y. El paciente respirador bucal: uma propuesta para el estado Nueva Esparta, 1996-2001. Acta Odontol. 2004; 42(2):28-40.

\section{RECEBIDO EM: 27/10/2006}

ACEITO EM: 04/02/2008

Endereço para correspondência:

Rua 24 de março, 273

Serra Negra - SP

CEP: $13930-000$

Tel: (19) 38924479

E-mail: csigolo@yahoo.com.br
12. Sabatoski CV, Mauro H, Camago ES, Oliveira JHG. Estudo comparativo de dimensões craniofaciais verticais e horizontais entre crianças respiradoras bucais e nasais. J Bras Ortodon Ortop Facial. 2002; 7(39):246-57.

13. Marchesan I. Avaliação e terapia dos problemas de respiração. In: Marchesan IQ, organizador. Fundamentos em fonoaudiologia: aspectos clínicos da motricidade oral. 2. ed. Rio de Janeiro: Guanabara Koogan; 2003. p. 23-6.

14. Coelho MF, Terra VHTC. Implicações clínicas em pacientes respiradores bucais. Rev Bras Patol Oral. 2004; 3(1):17-9.

15. Andrade FV, Andrade DV, Araújo AS, Ribeiro ACC, Deccaz LDG, Nemr K. Alterações estruturais de órgãos fonoarticulatórios e más oclusões dentárias em respiradores orais de 6 a 10 anos. Rev CEFAC. 2005; 7(3):318-25.

16. Foroni GBC, Aguirre V, Frisicaro CE. Persistencia de los movimientos primarios linguales: incidencia en la articulación del habla. [monografia]. Córdoba (Argentina): Escola de Fonoaudiologia da Faculdade de Ciências Médicas da Universidade Nacional de Córdoba; 1996.

17. Jury SC. Incidencia de los trastornos deglutorios funcionales en pediatria. Rev Chil Fonoaudiol. 2004; 5(1):7-20.

18. Castillo-Morales R, Molina G. Estúdio comparativo sobre la persistência de movimentos primários de lengua, en grupo de niños de cinco años con y sin compromiso neurológico. Rev Kinder de la Cátedra de Medicina Social de la Universidad de Munchen, Alemanha; 1995.

19. Molina G. El síndrome de Down en comunidades aborígenes argentinas: sus manifestaciones orofaciales [doutorado]. Córdoba (Argentina): Faculdade de Odontologia da Universidade Nacional de Córdoba; 1998.

20. Castillo-Morales R. Terapia de regulação orofacial. São Paulo: Memmom; 1999. 195 p.

21. El Hage SM. Avaliação alimentar em pacientes com paralisia cerebral: proposta fonoaudiológica. Rev Cienc Méd. 2001; 10(2):57-63.

22. Amaral CSF, Martins ER, Rios JBM. A respiração bucal e o desenvolvimento do complexo dentofacial. Rev Bras Alerg Imunopatol. 2002; 25(4):131-5.

23. Nieto Perea P, Acosta Quiñones JM, Meneses Lopes A. Determinación de la profundidad del paladar en niños com respiración bucal de 6-8 anõs de edad. Rev Estomatol Hered. 2005; 15(1):50-3.

24. Flutter J. The negative effect of mouth breathing on the body and development of the child. Int J Orthod. 2006; 17(2):31-7.

25. Silveira MC, Sígolo C, Quintal M, Sakano E, Tessitore A. Proposta de documentação fotográfica em motricidade oral. Rev CEFAC. 2006; 8(4):485-92. 